\title{
A case study of photon detection after acupuncture stimulation
}

\author{
Pagliaro G', Lauro $V^{2}$, Roversi $\mathrm{F}^{3 *}$ and Gullà $\mathrm{D}^{4}$ \\ ${ }^{1}$ Director of the Unit of Hospital Psychology, Oncologic Department, Bellaria Hospital, AUSL Bologna, Italy \\ ${ }^{2}$ Medical Doctor expert in Acupuncture, Medical Director of the Private Medical Clinic DLABMED, Bologna, Italy \\ ${ }^{3}$ Psychology Trainee, Bellaria Hospital, AUSL Bologna, Italy \\ ${ }^{4}$ Forensic Expert and Researcher at the Technologic Center and New Technologies Incubator ELDORLAB - CNR of Bologna, Italy
}

\begin{abstract}
Scientific literature highlighting forms of luminescence during an acupuncture treatment is very limited. In order to prove the functional reality of meridians, some contributions have tested infrared images, while others have used the inoculation of radioactive tracers. This pilot study aims at investigating whether the photon emission of the human organism (biophotons) may vary following the insertion of needles on specific points of the body (acupoints). In particular, the main objective of this case study is to verify whether the stimulation of certain acupoints in an healthy subject can elicit a variation in the photon emission (bioluminescence) from the body of the subject, within a 30-minute time frame, in the UV-VIS-IR spectral band, as documentable by the MIRA- (Hyperspectral Imaging "HIS") device. The secondary objective is to measure the variation of photon emission during a meditative practice performed by both the subject undergoing acupuncture and the acupuncturist. Photon detections performed after the infission of needles showed a clear incremental change in the flow of photons within the interested area of the subject's body, showing a correlation between biophotonic activation and the area stimulated through acupuncture. Moreover, this photon activation expands to the surroundings when both the acupuncturist and the subject start a meditation practice, with flows of photons moving from the acupuncturist to the subject as per the content of the performed visualization practice. Other relevant variations in the photon emission were measured, suggesting the hypothesis of an overall better homeostasis, a lower energetic consumption and a better oxygenation in the subject's body.
\end{abstract}

\section{Introduction}

Acupuncture is an ancient Chinese therapeutic technique, whose origin is dated back to 4,000 years ago. According to ancient Chinese texts, the insertion of needles on the skin surface of an individual determines an energetic variation until a state of balance in the body is achieved. Energy (Qi) is believed to flow along the body through a complex network of channels called "meridians". Scientific literature highlighting forms of luminescence during an acupuncture treatment is very limited. Over time, several attempts have been made in order to prove the functional reality of meridians through infrared images that could capture body energy tracing the paths of the main meridians [1-5]. Other attempts have used inoculation of radioactive tracers in acupoints or in random skin points, coming to the conclusion that the diffusion of the tracer would only start from acupoints. Among these contributions, a very interesting study describes the effects of Tc $99 \mathrm{~m}$ diffusion when injected in acupuncture points and in points outside the meridian [6]. This study was carried out by injecting a radioisotope, the technetium pertechnetate, both in an acupuncture point (LI4 Large Intestine Meridian, point 4) and in another point outside the acupuncture meridian. Results have highlighted the rapid migration of the radioisotope when this was injected in the acupoint, contrary to its stagnation when the injection was far from the acupoint. The study showed, already at that time, an unspecified anatomic-functional structure, which allowed the tracer to rapidly migrate along the theoretical path of acupuncture meridians without a correspondence with the pathways of the lymphatic, nervous and vascular systems.

The "MIRA-device" system, used in our pilot study, has been adopted with acupuncture in a previous study, during which a needle was inserted on a finger of the left hand, with the aim of stimulating the Large Intestine channel. After 15 minutes, a structure like a dotted channel, slightly brighter and more emissive, was detected by the device.

This pilot study, conducted on a single case, aims at investigating whether the photon emission of the human organism (biophotons) may vary following the insertion of needles on specific points of the body (acupoints). In particular, the main objective of this case study is to verify whether the stimulation of certain acupoints in an healthy subject can elicit a variation in the photon emission (bioluminescence) from the body of the subject, within a 30-minute time frame, in the UV-VIS-IR spectral band, as documentable by the "MIRA-device". The secondary objective is to measure the variation of photon emission during a meditative practice performed by both the subject undergoing acupuncture and the acupuncturist, with a specific and previously agreed timing (from minute 15 to minute 30).

\section{Materials and methods}

The experiment took place inside a facility with a protected environment and with levels of temperature and humidity oscillating within $2 \%$.

The detection and the energetic analysis were performed with a Hyperspectral Imaging (HSI) device, called "MIRA-device". The system

${ }^{*}$ Correspondence to: Francesca Roversi, Psychology Trainee, Bellaria Hospital, AUSL Bologna, Italy, E-mail: francesca.roversi@gmail.com

Received: May 29, 2020; Accepted: June 16, 2020; Published: June 22, 2020 
uses a multispectral sensor and an artificial intelligence that, in addition to algorithms, allow to detect minuscule variations in terms of intensity, chroma and flow stage of the photons reflected by the experiment participants.

The sensor works at 10 bit $\ 1024$ levels, but the analysis algorithms operating in binning are at 8 bit $\backslash 256$ levels. Levels from 0 to 128 represent negative variations compared to a $\mathrm{T}$ point of reference, while levels from 128 to 255 are positive variations.

In the following pages, some frames drawn from the video sequences of the experiment are shown. Some images are presented in succession, in order to highlight changes compared to the initial starting point. During the test, only one volunteer subject was measured. The energetic points were measured before the infission of needles (baseline) and then every 5 minutes, until minute 20. Lastly, a biofield measurement was performed at minute 30, after removing needles (Figure 1).

Once the subject positioned himself supine, the actupuncturist inserted 4 needles (TEWA, $0.20 \times 25 \mathrm{~mm}$ ) on the following acupoints (Figure 2): right and left Hegu LI4 (Large Intestine meridian, point 4), right and left Taichong LR3 (Liver meridian, point 3). The choice of these 4 points had the purpose of mobilizing as much as energy as possible, considering that in Chinese Medicine these points together are referred to as the "Four Gates": "Together, these points work to circulate the qi and blood through the body. They help to open all the meridians, increase circulation, and decrease pain anywhere in the body. The 4 Gates can also be used for emotional issues as well such as feelings of being trapped or stuck in a situation. This is a great point combination to help maintain the free flow of qi and blood through our bodies".

In Figure 2, detected after 20 minutes from needles infission (right picture), it is possible to observe the activation of 4 red macro-areas (arrows), in line with each other, starting from the right foot and reaching up to the anterior part of the right leg.

Approximately halfway through the experiment, after 15 minutes from needles infission, the acupuncturist, who was seating in close proximity to the feet of the subject (therefore not framed) on his right side, started a meditation practice. The subject started meditating at the same time. According to the meditative practice performed by the acupuncturist, she has imagined to send her own energy to the subject's body.

After 25 minutes from the infission of needles (meaning 5 minutes after both the acupuncturist and the subject started meditating), a very intense green/red coloring appears (Figure 3 ). This coloring comes from the right upper corner of the room (where the acupuncturist is seated; right arrow in Figure 3) and, simultaneously, a more intense red line appears in correspondence with the anterior area of the volunteer's right leg (left arrow in Figure 3) (Figure 4).

\section{Discussion and conclusions}

The study of the photon emission of biological organisms (biophotons) consists in detecting wavelengths of light not visible to the human eye, emitted by living beings, be they plants or animals. After the infission of needles, detections were made with the "MIRA-device" system, which showed a clear incremental change in the flow of photons within the framed area of the subject's body, namely the right foot and leg. Reminding the fact that in the lower limb the needles had been placed in the LR3 acupoint, the areas of biophotonic activation appear to be corresponding to the acupoints located on the Liver meridian (LR). Moreover, in a less clear way, an energetic activation seems to appear also on the same meridian of the left foot.

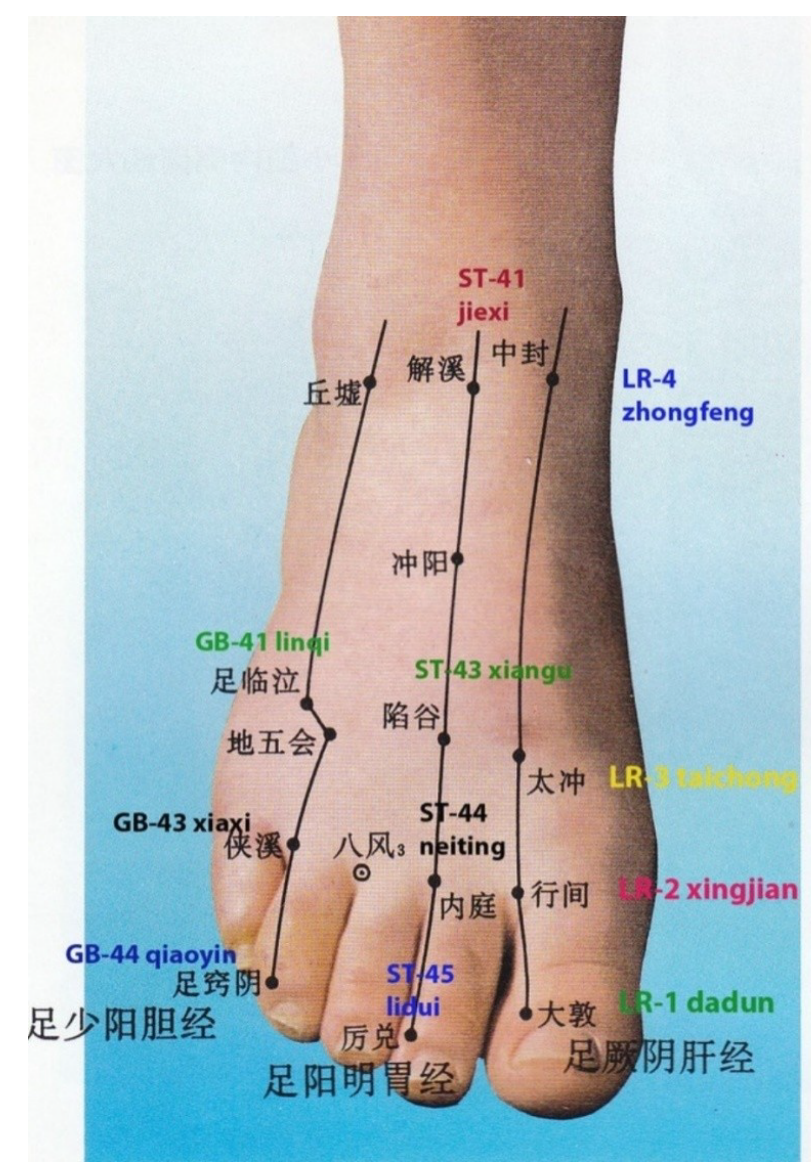

Figure 1. Map of the acupuncture points of the foot (Meridians and Acupoints)

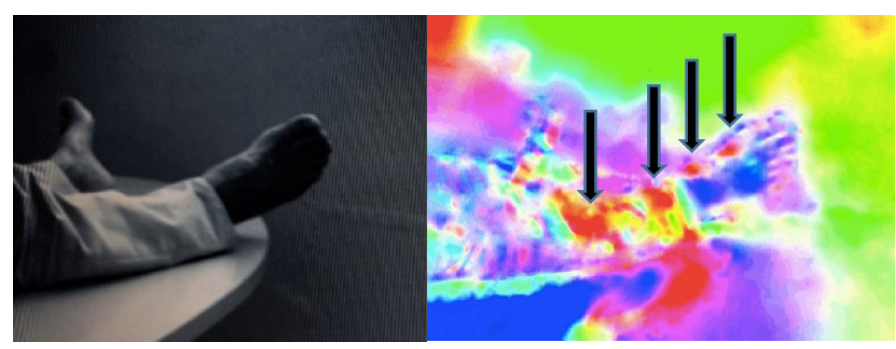

Figure 2. Energetic images detected with the "MIRA device" 20 minutes from needle infission

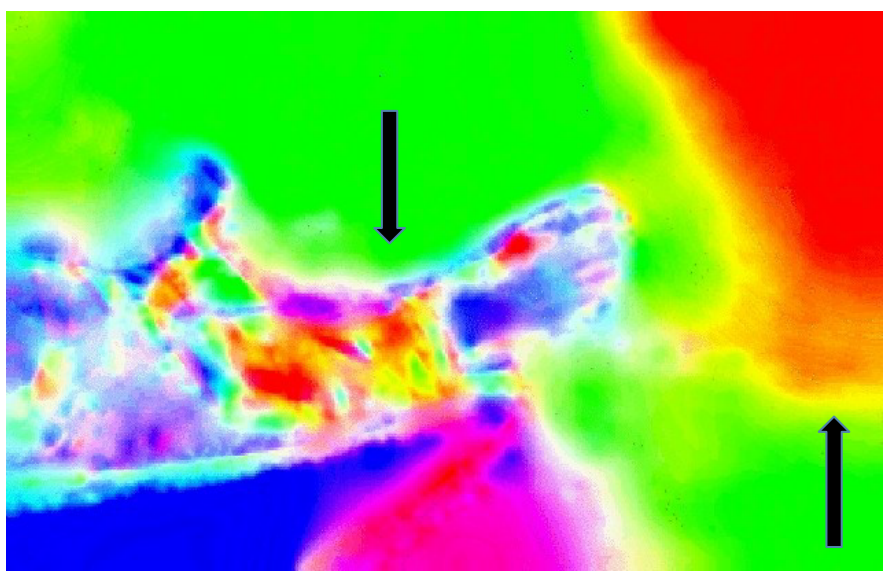

Figure 3. Energetic image detected after 25 minutes from needles infission 


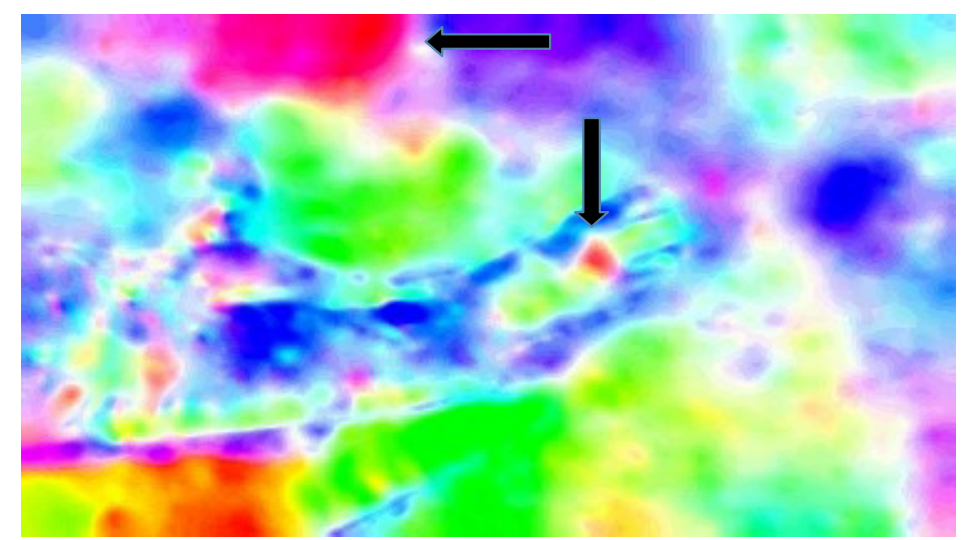

Figure 4. Frame at minute 30, right after removing the needles from the skin

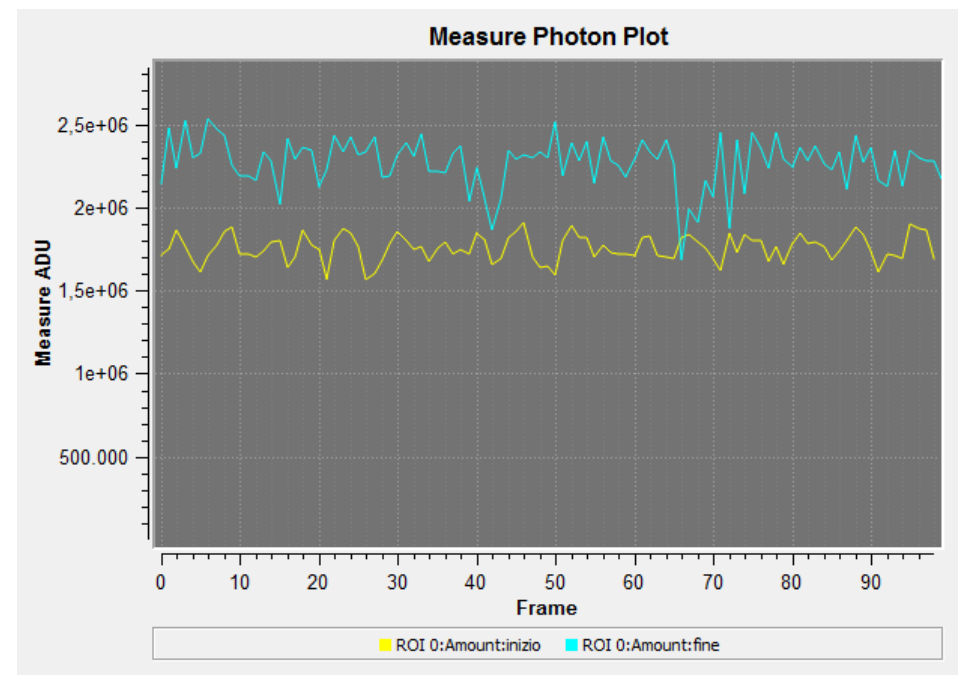

Figure 5. Trends of detected photons at the beginning (yellow line) and at the end (light blue line) of the experiment, measured on a small area of the right foot

From minute 15, the variation of energy emission over time becomes noticeable also in the area surrounding the subject. In fact, starting from that moment, the acupuncturist and the subject started meditating together. Differently from the subject, the acupuncturist was performing a visualization technique, which consisted in sending her own energy to the subject. From that moment, frames show an area with a more intense luminescence coming from the right side of the room, area where the acupuncturist was seating (not framed). From this area, it was possible to detect a flow of photons moving towards the subject's body, as per the content of the performed visualization where energy was in fact sent to the subject. During these phases it was also possible to notice an amplified brightness in the subject's areas of the right foot and leg. Theoretically, there could be a correlation between events: one could assume that the flow of photons coming from the acupuncturist and the one emitted by the subject (in this case both upon stimulation with acupuncture and meditation) create a single cumulative area of photons. This hypothesis will need further verifications through additional testing.

Another significant piece of data is represented by the fact that, although in some stages of the test an increased energy could be observed (and this phenomenon is usually associated to an increase of the photons' state of entropy, namely increased chaos), at the end of the experiment (minute 30) the global photonic entropy resulted to be 3 times lower than the initial entropy (baseline). This could suggest a better homeostasis in the subject and a lower energetic consumption for his habitual needs. The flow of photons in the blood, measured on a peripheral superficial level, proved to be the $50 \%$ lower in terms of variability, while the number of photons has increased. This could suggest a lower energetic consumption and a better oxygenation.

The quantity of emitted photons was compared between the start (before the infission of needles) and the end (after removing needles) of the treatment by assessing a small area of the right foot. At the beginning, the detected photons were 1.8 millions (yellow line in Figure 5), measured on a ROI of 10 square Pixel, while at the end of the test 2.5 millions photons (light blue line in Figure 5) were measured on the same ROI of 10 square Pixel. The increase in the flow of photons in the measured area has increased by $30 \%$.

\section{References}

1. Hu X, Wu B, Wang P (1993) Displaying of meridian courses travelling over human body surface under natural conditions. Zhen ci Yan Jiu 18: 83-89.

2. Kwang-Sup S (2003) Bonghan duct and acupuncture meridian as optical channel of biophoton. Journal of the Korean Physical Society 45: 1196-1198.

3. Slawinski J, Gorski Z (2008) Imaging of biophoton emission from electrostimulated skin acupuncture point jg4: Effect of light enhancers. Indian Journal of Experimental Biology 46: 340-344.

4. Hobbs R (2009) The biophysics of acupuncture: emerging patterns from selected studies. Medical Acupuncture 21. 
Pagliaro G (2020) A case study of photon detection after acupuncture stimulation

5. Chen GZ, Xu YX, Wang YH, Yang HQ, Lin QY, et al. (2011) Optical transport properties along the pericardium meridian under different pressure. J Lasers Med Sci 2: 89-97.
6. Giovanardi CM, Lonardo E, Abbati A(1999) Studio della diffusione del TC99m iniettato in un punto di agopuntura ed in un punto fuori meridiano. Rivista Italiana di Medicina Tradizionale Cinese 75: 20-21.

Copyright: (C2020 Pagliaro G. This is an open-access article distributed under the terms of the Creative Commons Attribution License, which permits unrestricted use, distribution, and reproduction in any medium, provided the original author and source are credited. 